\section{STAPLER DESIGN AND STRICTURES AT THE ESOPHAGOGASTRIC ANASTOMOSIS}

An apparent reduction in the rate of benign anastomotic stricture after stapled esophagogastrectomy prompted us to review the results obtained with different stapling devices since 1988 . We present a retrospective review of 125 consecutive patients undergoing esophageal resection for malignancy with stapled intrathoracic anastomoses. Benign anastomotic stricture was deemed present when a patient required endoscopic dilatation to treat postoperative dysphagia. We found no difference in risk factors not related to stapler size (tumor histologic characteristics, adjuvant therapy) between patients with stricture and patients without stricture. Event-free survival was compared for different stapler diameters as well as for different stapler designs. We found that staplers of smaller diameter were associated with significantly more strictures $(p<0.005)$. In a comparison of different designs of $25 \mathrm{~mm}$ stapler, the newer CDH device (Ethicon Ltd., Edinburgh, United Kingdom) was associated with a similar stricture rate to that associated with other designs (ILP [Ethicon] and EEA [Autosuture Company Division, United States Surgical Corp., Norwalk, Conn.]). For a given stapler diameter, it appears that different stapler designs have no effect on stricture rate. (J THORAC CaRdiovasc SuRg 1996;111:142-6)

R. G. Berrisford, BSc, ChM, FRCS, R. D. Page, MCh, FRCS, and

R. J. Donnelly, FRCSEd, Liverpool, England t is now recognized that stapled and hand-sewn intrathoracic esophagogastric anastomoses carry equally low leakage rates. ${ }^{1}$ Benign anastomotic stricture is more common, however, after stapled anastomoses. $^{1,2}$

This article evaluates the effect of stapler diameter and stapler design on development of anastomotic strictures. In our previous series of intrathoracic anastomoses made with EEA staplers (Auto Suture Company Division, United States Surgical Corp., Norwalk, Conn.), ${ }^{3}$ benign anastomotic stricture occurred in $9.5 \%$ of patients who underwent esophageal resection for cancer. Other authors report higher stricture rates of $20 \%$ to $30 \%,{ }^{1,2,4}$ but they do not quote separate rates for different stapler diameters. 1,2

We previously found that anastomotic narrowing was more common with staplers of smaller diame-

From the Cardiothoracic Centre, Liverpool, England.

Received for publication July 25, 1994.

Accepted for publication May 10, 1995.

Address for reprints: R. G. Berrisford, BSc, ChM, FRCS, Senior Registrar, The Cardiothoracic Centre, Thomas Dr., Liverpool, L14 3PE, England.

Copyright (c) 1996 by Mosby-Year Book, Inc.

$0022-5223 / 96 \$ 5.00+0 \quad \mathbf{1 2 / 1 / 6 6 2 3 0}$ ter, but these differences were not statistically significant. Griffin and colleagues ${ }^{4}$ have shown a significantly higher stricture rate with the EEA25 stapler compared with the EEA28 according to $\chi^{2}$ $(p<0.001)$. Because the number of patients at risk for stricture formation is not constant, event-free survival is a more appropriate means of comparing stricture rates among different groups.

During the past 2 years, we initially noticed a reduction in incidence of anastomotic strictures coinciding with our use of the newly designed $\mathrm{CDH}$ stapler (Ethicon Ltd., Edinburgh, United Kingdom). This prompted us to review our stapled esophagogastric anastomoses completed since 1988 with respect to event-free survival to investigate the effect of stapler design on anastomotic stricture formation.

\section{Methods}

The case notes of all patients undergoing esophagectomy for malignant disease since 1988 were reviewed retrospectively. Only patients who had stapled intrathoracic anastomoses were included in the study $(n=125)$. We excluded one patient who required cervical esophagostomy after resection of an ischemic stomach shortly after she underwent primary anastomosis. The study population comprised 124 patients; mean age was 63.7 years, age range was 35 to 85 years, and sex ratio (male to female) was 88:36. Tumor histologic classification was 


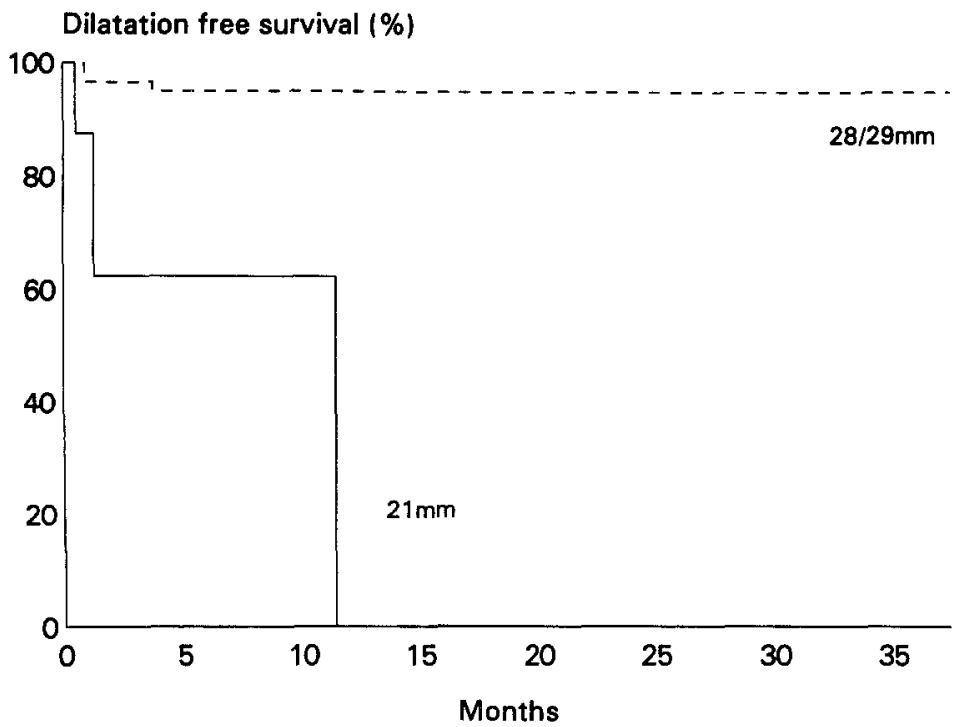

\begin{tabular}{lcccccc} 
& 30 days & 6 months & 1 year & 2 years & 3 years & 4 years \\
\hline $21 \mathrm{~mm}$ & 9 & 7 & 3 & 2 & 2 & \\
$28 / 29 \mathrm{~mm}$ & 33 & 29 & 22 & 9 & 6 & 3
\end{tabular}

Fig. 1. Kaplan-Meir event-free survival curves for patients with $21 \mathrm{~mm}$ and 28 or $29 \mathrm{~mm}$ anastomoses. The table shows the numbers of patients followed up with time. Log-rank analysis of the two survival curves yields $p<0.005$.

Table I. Anastomotic staplers and occurrence of benign anastomotic stricture

\begin{tabular}{|c|c|c|c|c|c|c|c|c|c|}
\hline & \multicolumn{3}{|c|}{$E E A$} & \multicolumn{3}{|c|}{$\overline{L L P}$} & \multicolumn{3}{|c|}{$C D H$} \\
\hline & \multirow[b]{2}{*}{$n$} & \multicolumn{2}{|c|}{ Strictures } & \multirow[b]{2}{*}{$n$} & \multicolumn{2}{|c|}{ Strictures } & \multirow[b]{2}{*}{$n$} & \multicolumn{2}{|c|}{ Strictures } \\
\hline & & No. & $(\%)$ & & No. & $(\%)$ & & No. & $(\%)$ \\
\hline $21 \mathrm{~mm}$ & 1 & 0 & 0 & 8 & 4 & 57 & - & - & - \\
\hline $25 \mathrm{~mm}$ & 7 & 2 & 29 & 44 & 11 & 27 & 24 & 3 & 14 \\
\hline $28 * / 29 \mathrm{~mm}$ & 17 & 0 & 0 & 13 & 2 & 17 & 7 & 0 & 0 \\
\hline $31 * / 33 \mathrm{~mm}$ & 2 & 0 & 0 & 1 & 0 & 0 & - & - & - \\
\hline
\end{tabular}

Stricture rate $(\%)$ calculated after excluding in-hospital deaths.

*EEA used came in 28 and $31 \mathrm{~mm}$ sizes, versus 29 and $33 \mathrm{~mm}$ for ILP and CDH devices.

adenocarcinoma in 73 patients $(58.8 \%)$, squamous carcinoma in 49 patients $(39.5 \%)$, and anaplastic carcinoma in two patients $(1.7 \%)$.

Whenever possible, we performed esophagectomy through a left thoracophrenotomy according to a technique we have described elsewhere. ${ }^{5,6}$ The largest stapler that would comfortably fit into the esophageal lumen was chosen. A purse-string suture was passed into the esophagus through a reusable purse-string clamp, and stay sutures were inserted to provide traction on four quadrants of the esophagus. We inserted the stapling instrument into the stomach through a vertical gastrotomy and passed it into the proximal esophagus without previous dilatation. The anastomosis was checked externally and through the gastrotomy after the spent stapler was re- moved. Extra sutures of 3-0 Mersilene (Ethicon, Inc., Somerville, N.J.) were inserted into the anastomosis if necessary. Any problems encountered in making the anastomosis were recorded in the operation note.

Patients were followed up at the thoracic surgical outpatient clinic at 6 weeks, 3 months, and 6 months after operation and thereafter at 6-month intervals. Any patient reports of dysphagia were investigated with fiberoptic endoscopy. If a stricture was present, it was sampled for biopsy and dilatated with Maloney bougies. Further dilatations were performed only if symptoms persisted.

Patient data were collated with PATS software (Patient Analysis and Tracking System; Dendrite Medical Systems, London, United Kingdom). Patients who died in the hospital ( $<30$ days after operation) or in whom anasto- 


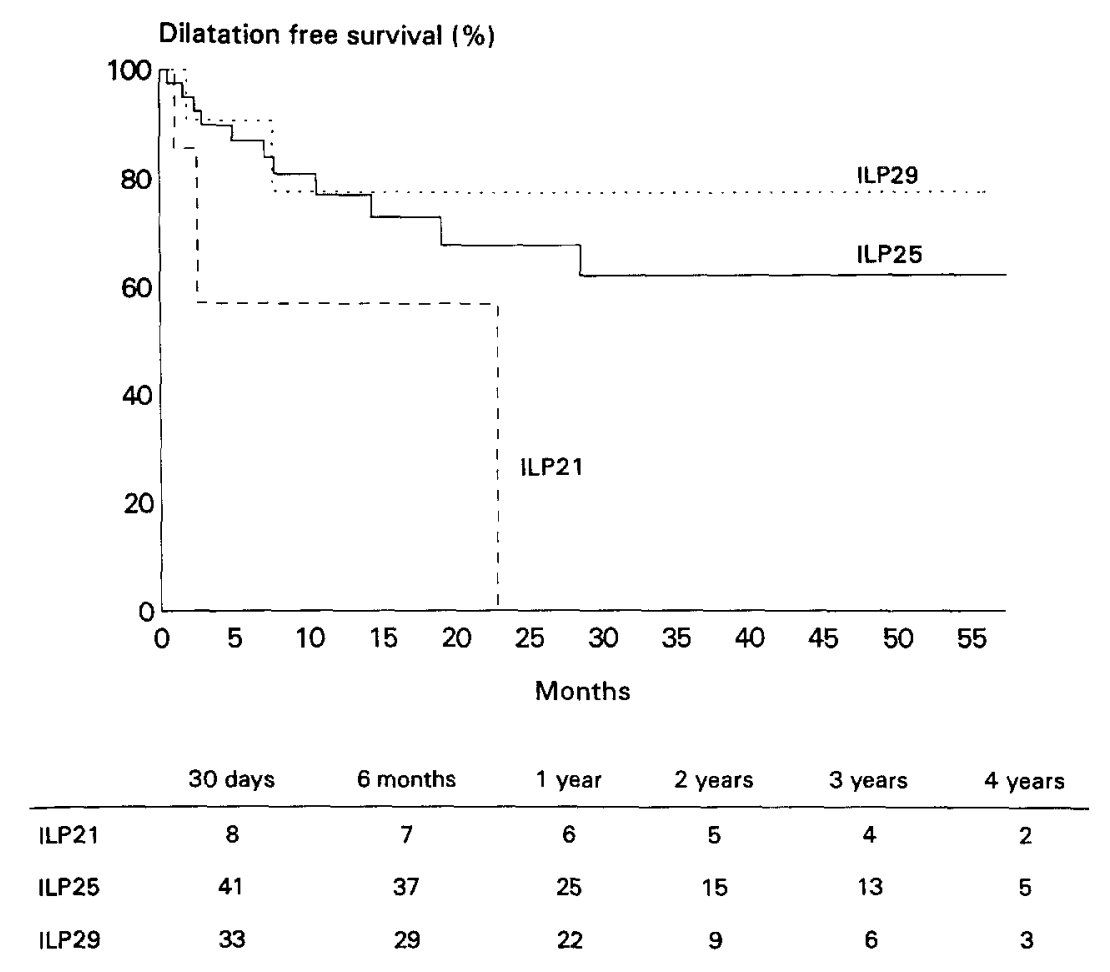

Fig. 2. Kaplan-Meier event-free survival curves for patients with anastomoses made with the ILP device. (21 mm, $25 \mathrm{~mm}$, and $29 \mathrm{~mm}$ diameters). The table shows the numbers of patients in each group at follow-up.

motic recurrence developed were excluded from calculations of stricture rate and event-free survival. KaplanMeier event-free survival curves were plotted for each stapler group, with "dilatation for benign stricture" defined as an event. Malignant stricture and normal findings at endoscopy were not deemed events. The log-rank test was used to determine the significance of difference between event-free survival curves.

\section{Results}

Follow-up was $98.5 \%$ complete (2609 months of a possible total of 2648 months). Hospital mortality rate was $8.0 \%(n=10)$. There were four anastomotic leaks (3.2\%), all of which were fatal. Anastomotic leakage was not associated with any particular type of stapler.

We have used three designs of stapler since 1988; EEA, ILP (Ethicon Ltd.), and CDH. The latter two are both marketed as intraluminal staplers. Table I lists the staplers with their relative stricture rates (calculated after excluding in-hospital deaths).

Stapler diameter. Postoperative strictures were most common in smaller anastomoses, particularly those made with $21 \mathrm{~mm}$ staplers. Stricture rate for all $21 \mathrm{~mm}$ staplers was 50\% (4/8), compared with $5.9 \%(2 / 34)$ for all 28 and $29 \mathrm{~mm}$ staplers. Comparison of stricture-free survival curves (Fig. 1) confirms this as a significant difference $(p<0.005)$. Fig. 2 illustrates the differences in dilatation-free survival between ILP21, ILP25, and ILP29 groups.

Stapler design. To isolate stapler design as a factor, we compared different designs of $25 \mathrm{~mm}$ stapler. There were fewer strictures in anastomoses made with the CDH device $(3 / 21,14.3 \%)$ compared with the ILP $(11 / 41,26.8 \%)$ and EEA devices $(2 / 7$, $28.6 \%$ ). Stricture-free survival curves for $\mathrm{CDH} 25$ and ILP25 groups are shown in Fig. 3. Although at initial follow-up there appeared to be fewer strictures in the CDH group, longer follow-up and event-free survival curves indicate that this differ ence is not significant.

Other risk factors for development of strictures. There were no significant differences between patients with and without stricture in terms of histologic characteristics, level of anastomosis, insertion of extra anastomotic sutures, or adjuvant 


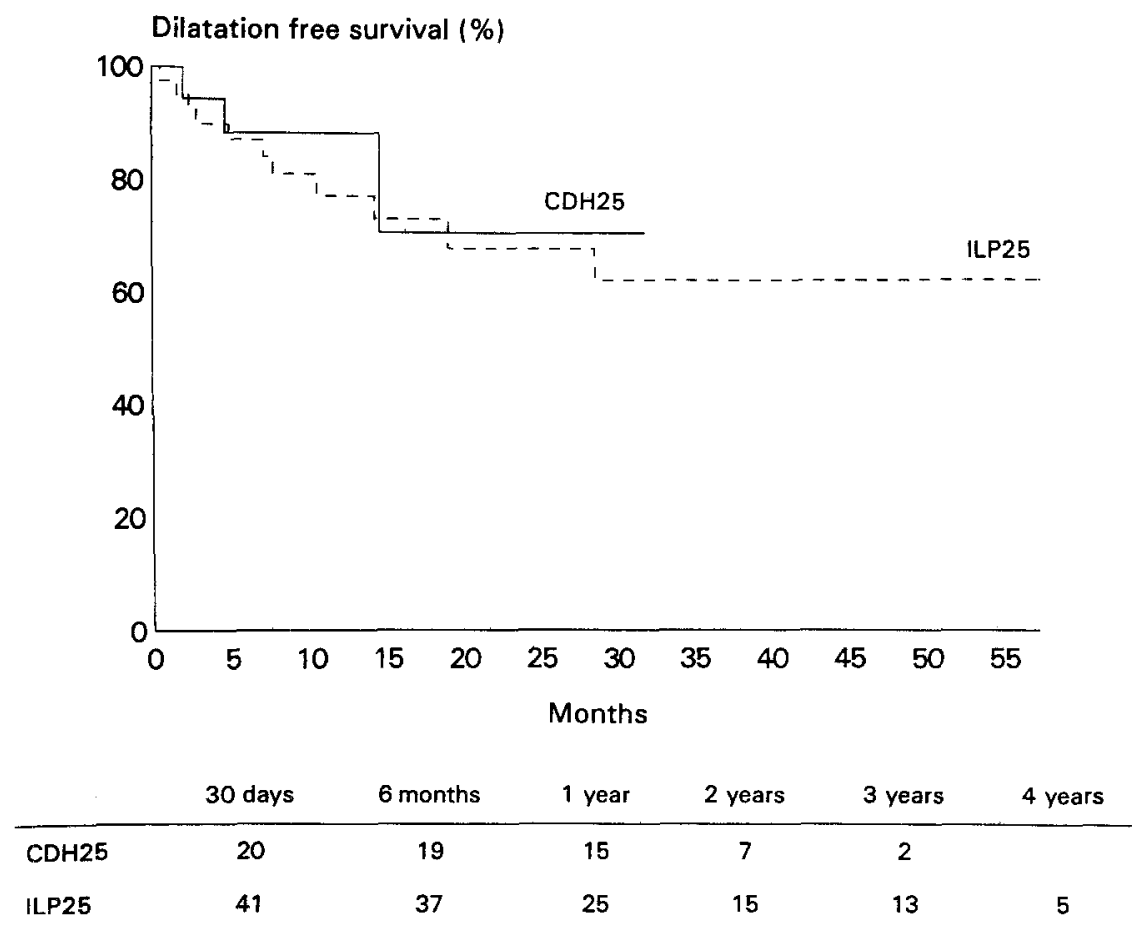

Fig. 3. Kaplan-Meier event-free survival curves for patients with anastomoses made with two $25 \mathrm{~mm}$ staplers of different design (ILP and CDH). The table shows the numbers of patients in each group at follow-up.

or neoadjuvant radiotherapy or chemotherapy (Table II). The level of anastomosis did not differ significantly between stapler groups.

Pattern of dilatation. Dysphagia occurred in 27 patients $(23.7 \%, n=114)$ during follow-up. Three of these patients did not have any stricture. Four had malignant anastomotic strictures, two of which arose de novo; the other two patients had previously undergone dilatation of benign strictures three times each. Median time to presentation of anastomotic stricture was 212.5 days (interquartile range 91 to 444 days, range 32 to 1280 days). Only four patients $(3.5 \%)$ required more than three dilatations. Operative treatment of benign anastomotic stricture was not necessary in any case.

\section{Discussion}

Have improvements in stapler design reduced the incidence of benign anastomotic stricture? We attempted to answer this question by comparing the different designs of $25 \mathrm{~mm}$ stapler. Although anastomoses made with the newer design $(\mathrm{CDH})$ were associated with a lower percentage of strictures, event-free survival curves revealed no true difference in stricture formation.
Table II. The prevalence of risk factors not related to stapler choice in patients with and without stricture (all staplers)

\begin{tabular}{lcc}
\hline & $\begin{array}{c}\text { Stricture-free group } \\
(n=94)\end{array}$ & $\begin{array}{c}\text { Stricture group } \\
(n=20)\end{array}$ \\
\hline Adenocarcinoma & 60 & 11 \\
Squamous carcinoma & 32 & 9 \\
Anaplastic carcinoma & 2 & 0 \\
Above/below arch ratio & $38: 56$ & $13: 7$ \\
Extra anastomotic sutures & 9 & 2 \\
Neoadjuvant radiotherapy & 8 & 3 \\
$\quad$ or chemotherapy & & 0 \\
Adjuvant radiotherapy & 9 & \\
$\quad$ or chemotherapy & & \\
\hline
\end{tabular}

What differences between the $\mathrm{CDH}$ and ILP staplers have been claimed might make a potential difference in stricture rate? The $\mathrm{CDH}$ anastomosis is made with titanium staples, whereas the ILP anastomosis is made with stainless-steel staples. Because it is a more inert metal, titanium may cause less tissue reaction and fibrosis. Access for tying the purse-string suture has been improved by introducing a detachable head and anvil shaft in the $\mathrm{CDH}$ stapler, and the body of the instrument is curved to 
improve approximation of stomach and esophagus without tension. To ease withdrawal of the spent CDH cartridge, the stapler drivers automatically retract into the cartridge housing and the stapler head is beveled. The knob for setting the staple gap has been made more sensitive, and the flag indicator (set at $2.0 \mathrm{~mm}$ on the ILP) has been updated to a continuous "green" range covering 1.0 to $2.5 \mathrm{~mm}$. This allows the surgeon to feel the force being used to clamp esophagus and stomach together, and allows tightening of the staples much as the surgeon would tighten a suture. The degree of tightening is well recognized as a critical point of technique.

Anastomoses made with the ILP25 and EEA25 instruments have already been shown to be associated with similar stricture rates. ${ }^{5,7,8}$ This suggests that differences in the diameter of anastomotic lumen (16.4 mm for ILP25 and $15 \mathrm{~mm}$ for EEA25), the thickness of anastomotic lip (1.77 $\mathrm{mm}$ for ILP25 and $2.02 \mathrm{~mm}$ for EEA25), and the number of staples per anastomosis (20 for ILP25 and 22 for EEA25) are not important as design features aimed at preventing anastomotic strictures.

We believe that it is important to inspect the anastomosis from within its lumen if leakage is to be avoided. We have shown that placement of additional sutures, which was necessary in 11 of 125 anastomoses, does not increase stricture formation. Indeed, competence of an anastomosis must never be jeopardized out of fear of causing a stricture. Further reduction in anastomotic lip size in future stapler designs, in particular, could lead to an increased risk of anastomotic leakage. In common with others, ${ }^{7-9}$ we found no differences between ILP25 and EEA25 anastomosis leakage rates. Anastomotic leakage rate was $3.2 \%$ in this series, remarkably consistent with rates in other series. ${ }^{1,4,5,8,9}$

The most important predictor of anastomotic stricture formation is the diameter of the anastomotic lumen. We demonstrated a highly significant differences in stricture-free survival with small and large staplers and a reduction in stricture rate with each successively larger size of ILP device. Staplers with smaller diameters have been associated with higher stricture rates in previous publications, ${ }^{4-6}$ but none of these studies showed significant differences in stricture-free survival. Strictures probably develop more frequently at smaller anastomoses because proliferation of granulation tissue narrows an already small lumen to a critical diameter associated with dysphagia. ${ }^{4}$

Significant postoperative dysphagia after esophagectomy occurs in about $20 \%$ of patients. ${ }^{6}$ It is a good predictor of anastomotic stricture, which was found in $88 \%$ of patients who underwent endoscopy for dysphagia. Two patients with anastomotic recurrence had already undergone three dilatations for apparently benign stricture, so repeated biopsy of apparently benign strictures is important.

Treatment of anastomotic stricture by dilatation maintained satisfactory swallowing in all the patients in this series. Anastomotic stricture remains a complication particularly associated with the stapled anastomoses $25 \mathrm{~mm}$ or less in diameter. Improvements in stapler design do not appear to have had much impact on this complication. Nevertheless, anastomotic stricture is a nuisance, rather than a life-threatening complication of esophagectomy.

\section{REFERENCES}

1. Lam TC, Fok M, Cheng SW, Wong J. Anastomotic complications after esophagectomy for cancer. J THORac Cardiovasc Surg 1992;104:395-400.

2. Fok M, Ah-Chong AK, Cheng SWK, Wong J. Comparison of a single layer continuous hand-sewn method and circular stapling in 580 oesophageal anastomoses. Br J Surg 1991;78:342-5.

3. Muehrcke DD, Kaplan DK, Donnelly RJ. Anastomotic narrowing after esophagogastrectomy with the EEA stapling device. J Thorac Cardiovasc Surg 1989;97: 434-8.

4. Griffin SM, Chan A, Chung SC, Li AK. Early and late surgical complications of subtotal oesophagectomy for squamous cell carcinoma of the oesophagus. J R Coll Surg Edinb 1991;36:170-3.

5. Muehrcke DD, Donnelly RJ. Complications after esophagogastrectomy using stapling instruments. Ann Thorac Surg 1989;48:257-62.

6. Page RD, Khalil JF, Whyte RI, Kaplan DK, Donnelly RJ. Esophagogastrectomy via left thoracophrenotomy. Ann Thorac Surg 1990;49:763-6.

7. Fekete F, Gayet B, Place S, Biagini J. Four hundred esophageal anastomoses with a stapler. Int Surg 1989; 74:69-72.

8. Wong J, Cheung H, Lui R, Fan YW, Smith A, Siu KF. Esophagogastric anastomosis performed with a stapler: the occurrance of leakage and stricture. Surgery 1987; 101:408-18.

9. Paterson IM, Wong J. Anastomotic leakage: an avoidable complication of Lewis-Tanner oesophagectomy. Br J Surg 1989;76:127-9. 